\title{
Error-prone polyploid mitosis during normal Drosophila development
}

\author{
Donald T. Fox, ${ }^{1,2}$ Joseph G. Gall, ${ }^{2}$ and Allan C. Spradling ${ }^{1,2,3}$ \\ ${ }^{1}$ Howard Hughes Medical Institute Research Laboratories, Carnegie Institution for Science, Baltimore, Maryland 21218, USA; \\ ${ }^{2}$ Department of Embryology, Carnegie Institution for Science, Baltimore, Maryland 21218, USA
}

\begin{abstract}
Endopolyploidy arises during normal development in many species when cells undergo endocycles-variant cell cycles in which DNA replicates but daughter cells do not form. Normally, polyploid cells do not divide mitotically after initiating endocycles; hence, little is known about their mitotic competence. However, polyploid cells are found in many tumors, and the enhanced chromosomal instability of polyploid cells in culture suggests that such cells contribute to tumor aneuploidy. Here, we describe a novel polyploid Drosophila cell type that undergoes normal mitotic cycles as part of a remodeling process that forms the adult rectal papillae. Similar polyploid mitotic divisions, but not depolyploidizing divisions, were observed during adult ileum development in the mosquito Culex pipiens. Extended anaphases, chromosome bridges, and lagging chromosomes were frequent during these polyploid divisions, despite normal expression of cell cycle regulators. Our results show that the switch to endocycles during development is not irreversible, but argue that the polyploid mitotic cycle is inherently error-prone, and that polyploid mitoses may help destabilize the cancer genome.
\end{abstract}

[Keywords: Polyploidy; endocycle; chromosomal instability; hindgut; Drosophila]

Supplemental material is available at http://www.genesdev.org.

Received May 27, 2010; revised version accepted August 20, 2010.

Endopolyploidy, the acquisition by cells of more than two complete sets of chromosomes, is common both during normal development and in tumors (Brodsky and Uryvaeva 1985). Vertebrate tissues-including liver, myocardium, uterus, placenta, and bone marrow-contain a significant number of polyploid cells. Many larval and adult tissues in insects such as Drosophila melanogaster use the endocycle (Lee et al. 2009) and are made up of predominantly polyploid cells. In these organisms, polyploidy is generally a late step in cellular differentiation. Once diploid cells switch to the endocycle, they can continue to grow in size and ploidy, but they rarely, if ever, return to mitotic division. Thus, mitotic cycles precede the endocycle, rather than vice versa.

Several observations suggest that polyploid cells are less suitable for mitosis. Cancerous tissue often contains polyploid cells and exhibits high levels of aneuploidy (Rajagopalan and Lengauer 2004). These observations, and the finding that experimentally induced tetraploid cells become chromosomally unstable (Ganem et al. 2007), have led to the proposal that polyploidy compromises genomic stability and contributes to oncogenesis. However, evidence linking polyploid mitosis to chromosome instability during normal development is lacking due to the absence of natural polyploid mitotic cycles.

${ }^{3}$ Corresponding author.

E-MAIL spradling@ciwemb.edu; FAX (410) 243-6311.

Article is online at http://www.genesdev.org/cgi/doi/10.1101/gad.1952710.
The transformation of a Drosophila larva into an adult fly during pupal development involves morphogenetic and cell cycle changes that are among the least-studied aspects of the life cycle. This is particularly true in the case of the adult hindgut, whose cellular precursors among larval gut and genital imaginal disc tissue have not been fully described (Skaer 1993). The major larval hindgut regions (Fig. 1A) - the sphincter-like pylorus, the absorptive ileum, and the water-transporting rectum (Berridge and Gupta 1967) - all undergo dramatic alterations, some of which were characterized recently (Fox and Spradling 2009). In the mosquito, polyploid cells of the larval ileum were reported to undergo somatic reduction divisions (i.e., sequential mitoses in the absence of DNA replication) to generate adult ileal cells of lower ploidy (Berger 1938). While investigating the metamorphosis of the larval Drosophila rectum, we discovered evidence of polyploid cells that are capable of mitotic division. Our results support the notion that polyploidy renders cells susceptible to mitotic abnormalities.

\section{Results}

\section{Cellular origin of the adult Drosophila rectum}

To look for pupal cells undergoing novel cell cycles, we first characterized the origin of the adult Drosophila rectum from the larval rectum and genital disc (Fig. 1A). We examined rectal and genital disc tissue at regular 


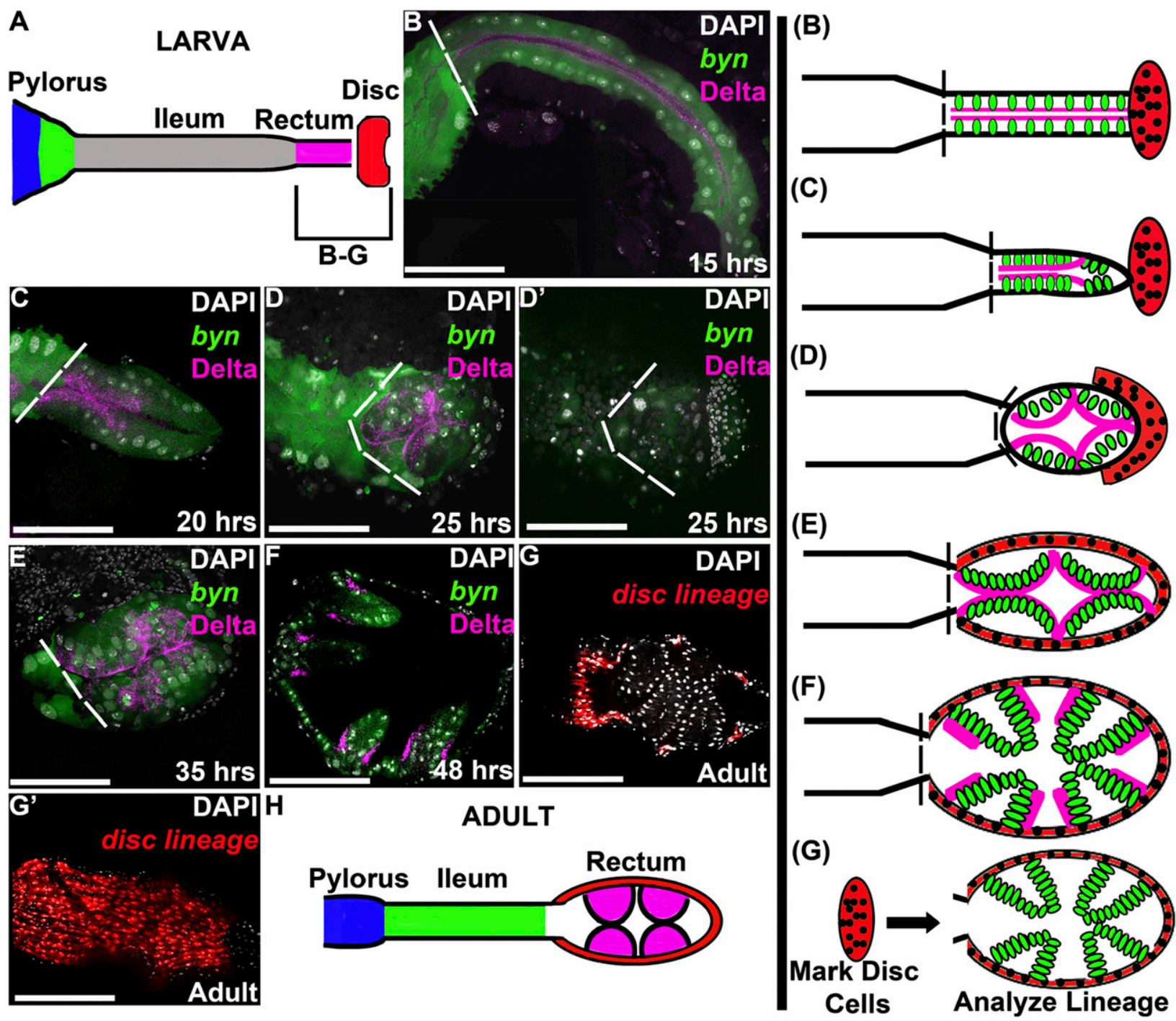

Figure 1. Rectal morphogenesis and growth during Drosophila metamorphosis. (A) Larval Drosophila hindgut. (Blue, green) Adult progenitor regions of the pylorus; (gray) ileum; (purple) rectum; (red) genital disc. (B-F) Adult rectal organogenesis. (Dashed line) Ileum/ rectum boundary. (Right column) Cartoons of indicated micrographs: (green) byn-labeled rectal nuclei; (purple) Delta in rectal lumen and along papillae; (red) genital disc. (B) Fifteen hours post-puparium formation. (C) Twenty hours post-puparium formation. Rectal cells begin rounding up. $(D)$ Twenty-five hours post-puparium formation. Genital disc cells (small nuclei at right in $D^{\prime}$, which is in a different focal plane from $D$ ) associate with rounding papillae. $(E)$ Thirty-five hours post-puparium formation. Disc-derived cells fully surround growing papillae. $(F)$ Forty-eight hours post-puparium formation. Papillar growth complete; distal papillar cells retain Delta. $\left(G, G^{\prime}\right)$ Genital disc cell lineage marked in larva by $d s x$-GAL4, then analyzed in the adult. $(G)$ Optical section showing that papillae are not disc-derived. $\left(G^{\prime}\right)$ Optical section showing that diploid rectal surface cells are disc-derived (red). (H) Adult hindgut, colored as in $A$ to illustrate the origin of adult regions from larval precursors. Bar, $100 \mu \mathrm{m}$.

intervals throughout pupal development and followed individual cell types by staining to reveal expression of brachyenteron (byn), encoding a hindgut-specific transcription factor, and the signaling molecule Delta (Fig. 1B-F, and right column). These studies provided a new general overview of rectal development. Most larval polyploid hindgut cells, such as those of the ileum, undergo apoptosis and are replaced by the division of mitotic progenitors (Fig. 1, A vs. H; Fox and Spradling 2009). However, larval rectal cells do not turn over, but increase in number in a process that generates the adult rectum with its four rectal papillae. The outer surface of the rectum, but not the papillae, derives from the genital disc (Fig. 1G), as shown by lineage marking larval genital disc cells using a genital disc Gal4 driver and FLP-out reporter (see the Materials and Methods).

Polyploid mitotic cycles during Drosophila and Culex hindgut development

To determine whether pupal rectal papillar cells increase in number via polyploid mitotic cycles, we first investigated whether they start out polyploid, as their large size suggests. We quantitated the level of DAPI staining in rectal nuclei prior to $(15 \mathrm{~h})$ and after $(52 \mathrm{~h})$ rectal remodeling, compared with sperm nuclei (Supplemental 
Fig. S1A). All rectal cells at the onset of pupation were 8C (Fig. 2A), while, after remodeling, cells at the narrow end of the papilla near the gut lumen remained $8 \mathrm{C}$, but distal cells increased to $16 \mathrm{C}$ (Fig. 2B).

The most striking indication that these polyploid rectal cells proliferate mitotically was the presence of cells with 32 metaphase chromosomes, the expected number for octoploid (8N) cells (Fig. 2C). Polyploid metaphases as well as phospho-histone $\mathrm{H} 3$ (PH3) staining were observed in rectal cells only during a precise window between $20 \mathrm{~h}$ and $48 \mathrm{~h}$ of pupal development (Fig. 2D). During this time, one could identify all stages of mitosis, including anaphase and cytokinesis, suggesting that these cells undergo complete mitosis, not endomitosis (Fig. 2E-I). Time-lapse movies of developing rectal papillar cells also strongly indicated that cytokinesis is complete following polyploid mitosis (Fig. 2J; Supplemental Movie S1). Finally, simple counts of rectal cell number before and after the mitotic period demonstrated that the mitotic cycle is complete. At $20 \mathrm{~h}$, only $\sim 100$ rectal cells are present, 25 in each nascent rectal papilla (Fig. 2K). However, by $48 \mathrm{~h}$, each papilla contains $\sim 100$ cells (Fig. $2 \mathrm{~K}$ ). Thus, every $8 \mathrm{C}$ precursor likely undergoes two complete mitotic divisions, after which the distal subpopulation undergoes one final endocycle to reach 16C. We concluded that polyploid rectal papillar precursors expand by mitotic division despite having already undergone two endocycles during larval development.

More than 70 years ago, the polyploid larval ileum of the mosquito Culex pipiens was reported to undergo somatic reduction divisions to form the adult ileum (Berger 1938). Consecutive divisions based on somatic chromosome pairing, without intervening $S$ phases, were believed to increase cell number but reduce cell ploidy. To compare hindgut development in Culex with the events just described in Drosophila, we looked for metaphase cells during pupal hindgut development in C. pipiens. During a narrow time window near the start of pupal development (Fig. 3A), large cells in prophase, metaphase, and anaphase that clearly contained many more than the six chromosomes expected of a diploid cell were observed in the ileum (Fig. 3B-D). However, we found no evidence that these divisions reduce cell ploidy. Ploidy levels in the ileum measured cytophometrically were 8C before (Fig. $3 \mathrm{E}, \mathrm{G})$ and $8 \mathrm{C}$ or $16 \mathrm{C}$ after the period when metaphase cells were present (Fig. 3F,H). Nonetheless, cell counts confirmed that the number of ileal cells increased during this same period (Fig. 3I). Rather than undergoing reductive divisions, our experiments suggest that, like Drosophila rectal cells, polyploid mosquito ileal cells re-enter the normal mitotic cycle.

\section{Regulation of rectal papillar endocycles and polyploid mitotic cycles}

Finding polyploid cells that proliferate via mitosis prior to final differentiation was surprising. Many Drosophila polyploid cells lack a late $S$ phase and consequently underreplicate their heterochromatin, a process expected to compromise centromere function and abolish mitotic
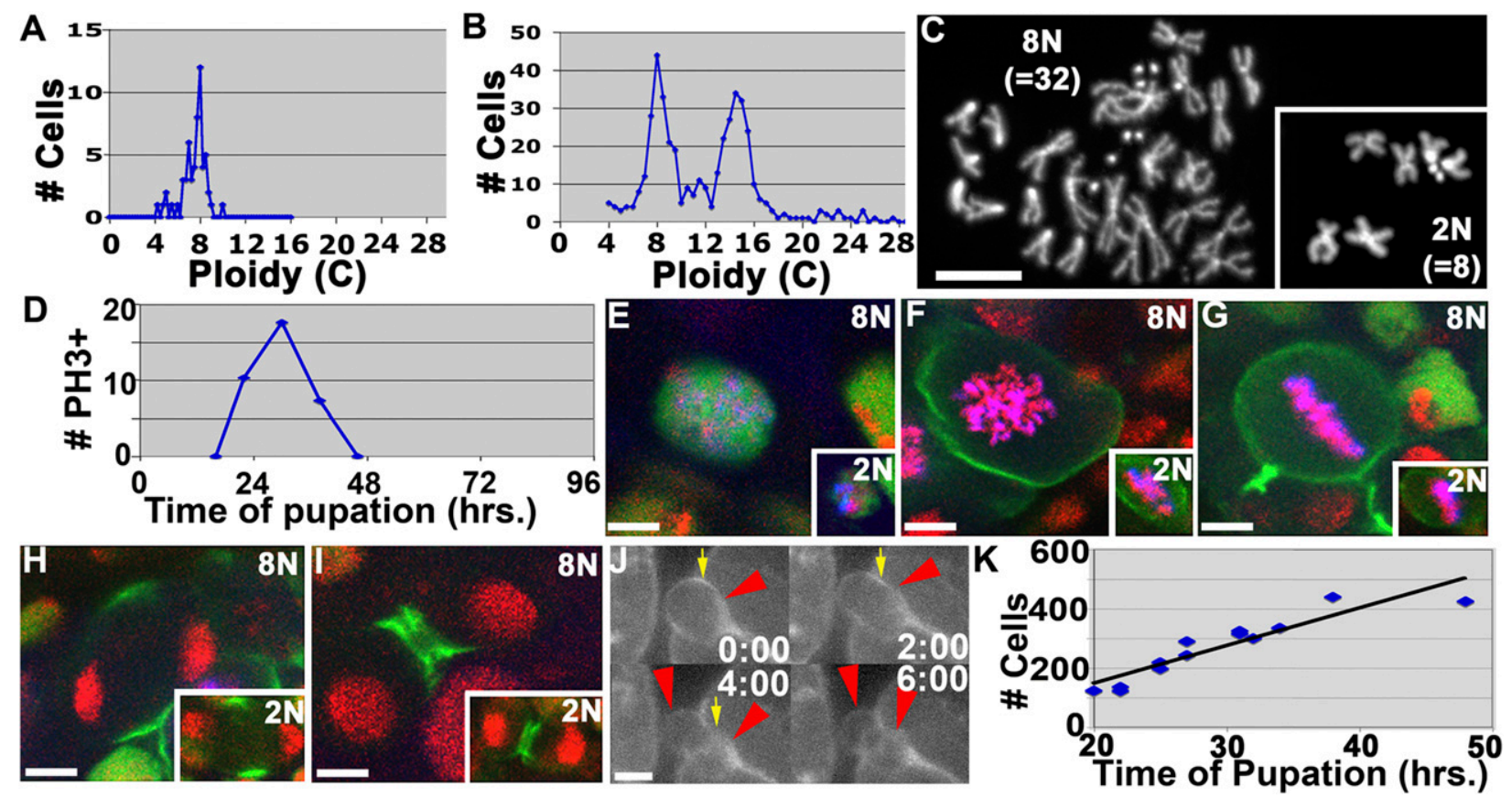

Figure 2. Polyploid mitoses in the Drosophila pupal rectum. $(A, B)$ Ploidy of rectal polyploid cells prior to $(A)$ or after $(B)$ gut remodeling. (C) DAPI-stained karyotype of $8 \mathrm{~N}$ polyploid and $2 \mathrm{~N}$ diploid (inset) mitotic pupal rectal cells. $(D)$ Graph of average number of $\mathrm{PH}^{+}$polyploid rectal cells during pupation. (E-I) Polyploid (and diploid; see insets) pupal rectal cells progress through mitosis. (Green) Anilin; (blue) PH3; (red) DAPI. PH3 expression diminishes after metaphase. $(E)$ Early prophase. $(F)$ Prometaphase. $(G)$ Metaphase. $(H)$ Anaphase. (I) Telophase. (J) Time-lapse analysis of cytokinesis using moesin::GFP to mark cell membranes. (Red arrowheads) Cells; (yellow arrows) cleavage furrows. Time is shown in minutes. $(K)$ Graph of total polyploid rectal cells during pupation. Insets are at the same scale as their parent panels. Bar, $5 \mu \mathrm{m}$. 

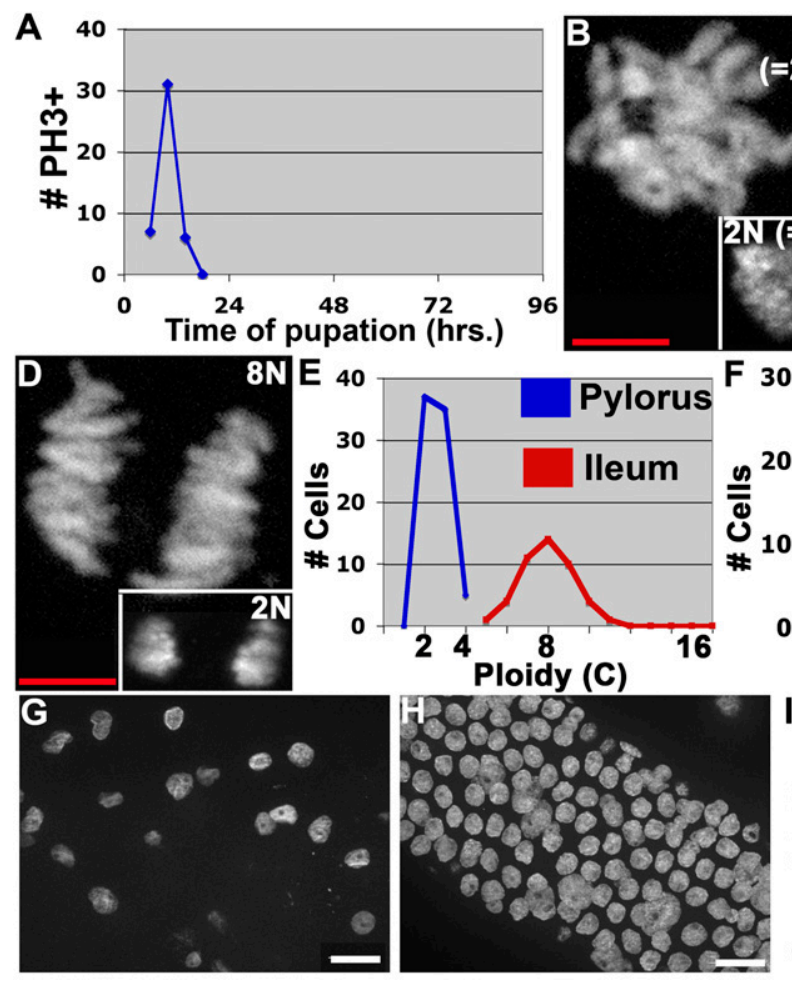
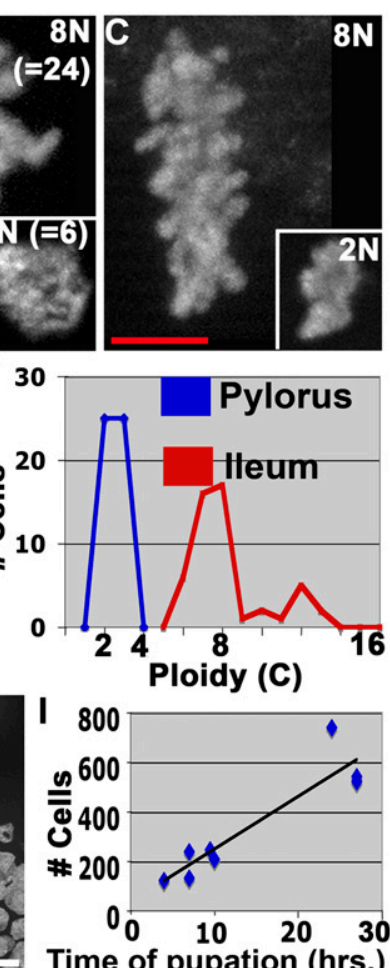

Figure 3. Polyploid mitoses in the Culex pupal ileum. (A) Graph of the average number of $\mathrm{PH}^{+}$polyploid cells in the Culex ileum as a function of time of pupation. $(B-D)$ PH3 labels diploid $(2 \mathrm{~N})$ and polyploid $(8 \mathrm{~N})$ mitotic cells of the Culex pylorus and ileum, respectively. $(B)$ Prophase. (C) Metaphase. (D) Anaphase. $(E, F)$ Ploidy analysis of the Culex pylorus (blue) and ileum (red) before $(E)$ and after $(F)$ the mitotic period. Relative DAPI intensity of pyloric and ileal cells does not decline following hindgut remodeling and mitosis. (G) DAPI staining shows the density and DNA content of Culex ileal cells before the mitotic period. $(H)$ DAPIstained Culex ileum after mitotic period, showing increased cell density and similar DNA content. $(I)$ Graph of the number of cells found in the Culex ileum of individual animals at the time of pupation indicated. Insets are at the same scale as their parent panels. Bars; red, $5 \mu \mathrm{m}$; white, $20 \mu \mathrm{m}$.

capability (Lee et al. 2009). However, ovarian nurse cells undergo four initial endocycles with a more complete S phase (Dej and Spradling 1999). To look for the presence of late S phase during the larval endocycles that produce the adult rectal papillar precursors, we labeled rectal cells in L2 larvae with the nucleotide analog EdU. In Drosophila, late replication can be recognized by incorporation that is confined to genomic heterochromatin, which aggregates within a small subnuclear region. Larval ileal cells in the same animals-cells that polyploidize extensively and do not divide again-provided a comparison. Ileal cells showed only early S-phase patterns of EdU incorporation into euchromatin throughout the nucleus (Fig. 4A,C), but rectal cells displayed both early and late $S$ patterns (Fig. 4B,C). The relatively high fraction of late $S$ patterns observed (Fig. 4C) likely reflects cell cycle synchronization prior to moulting (Graves and Schubiger 1982). Thus, rectal papillar precursor cells undergo larval endocycles with a late $S$ phase, and consequently replicate their genomes more fully than most endocycling Drosophila cell types. Nonetheless, replication may still be incomplete and might impair their ability to divide normally.

We looked for differences between the regulation of the polyploid mitotic cycles and other mitotic cell cycles. Polyploid mitotic $S$ phase showed normal characteristics. Cyclin E (CycE) levels fluctuate (Fig. 4D,G) and EdU is incorporated in both early and late phase patterns (Fig. 4E,G). Polyploid $M$ phase was also accompanied by a normal gene expression program. Cyclin A (CycA) expression transitioned from cytoplasm to nucleus at $\mathrm{G} 2 / \mathrm{M}$ (Fig. 4F, $\mathrm{F}^{\prime}, \mathrm{G}$ ) and was gone at metaphase (Fig. 4F"). This is in contrast to Drosophila endocycles, where CycA and other mitotic genes are not expressed (Lee et al. 2009). Telophase and cytokinesis were complete, based on staining of Anillin and Moesin-GFP (Fig. 2I,J) and the absence of binucleate cells (Fig. 4H). Confirming our ploidy analysis (Fig. 2B), we observed that the subset of papillar cells located distal to the gut lumen incorporate EdU after the mitotic period (Fig. 4I), supporting the view that a final endocycle increases their ploidy to $16 \mathrm{C}$.

We also examined whether cell cycle genes are functionally required for polyploid mitotic cycles. Using a hindgut-specific GAL4 driver (byn-GAL4) and a temperature-sensitive GAL80 repressor, RNAi transgenes targeting various cell cycle genes were expressed after larval rectal cells become $8 \mathrm{C}$ but prior to the onset of pupal mitotic cycles. We scored the number of rectal papillar cells in newly eclosed adults to determine whether mitotic proliferation of the 100 initial cells had been affected. Control animals exhibited the normal number of $\sim 400$ papillar cells (Fig. 5A,F), suggesting that, on average, each papillar precursor completes two rounds of division. In contrast, knockdown of the mitotic cyclin-dependent kinase $c d c 2$ or its phosphatase, stg, greatly reduced papillar cell number, indicating that these cell cycle regulators are required for the polyploid mitotic cycle (Fig. 5B,C,F). Similar results were seen for $C y c E$ and $C y c A$ (Fig. 5D,F). Overall, these experiments demonstrated that polyploid and diploid mitotic cell cycles operate in a similar manner.

We briefly examined the role of Notch signaling in the process of rectal papillar formation, since the Delta ligand was observed to accumulate on the apical surfaces of papillar cells (Fig. 1B-F). When a dominant-negative Notch transgene or Notch RNAi was expressed during pupal development, the number of rectal papillae that formed 

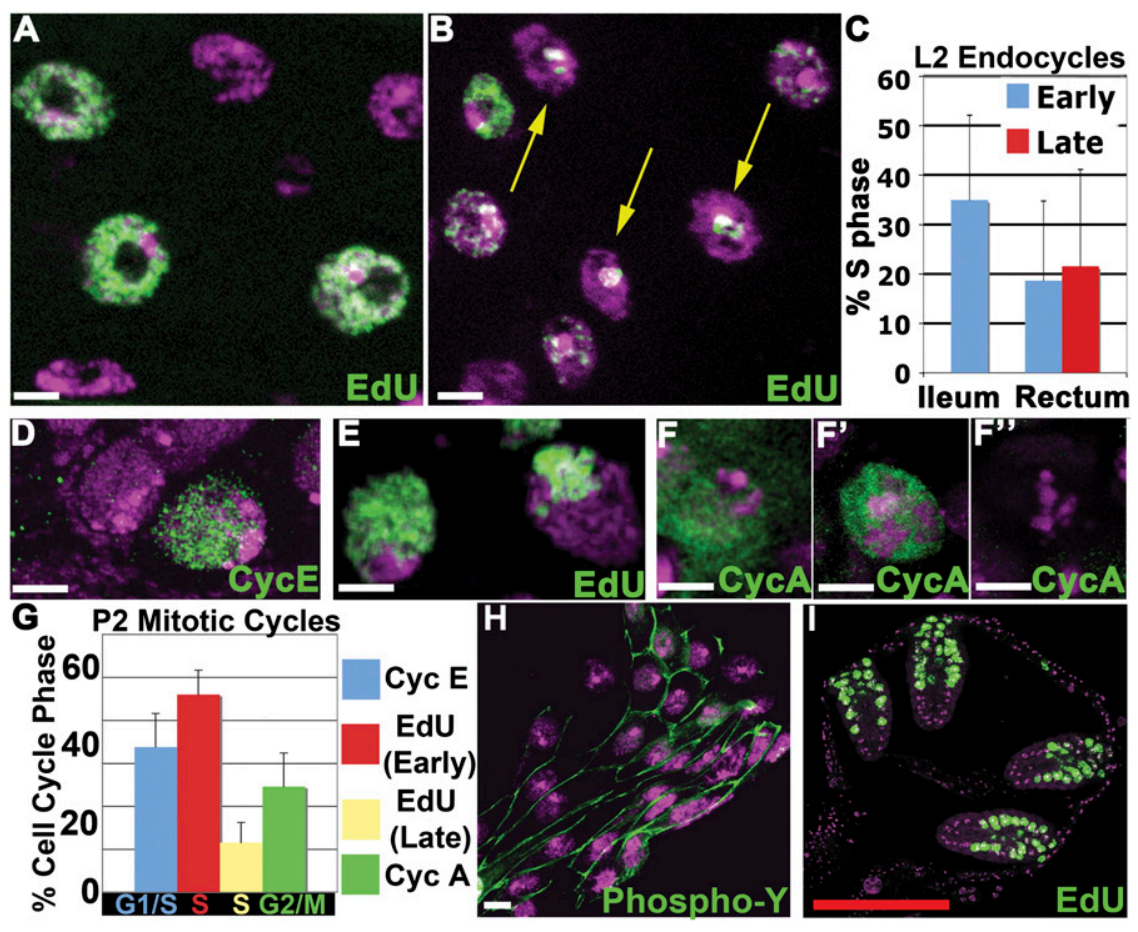

Figure 4. Cell cycle characteristics of polyploid mitotic cells. $(A-C)$ EdU labeling of endocycle $\mathrm{S}$ phases in second larval instar (L2) Drosophila hindguts. (A) Early $S$ patterns in ileal cells. (B) Early and late (yellow arrows) S patterns in rectal cells. (C) Quantitation of endocycle S-phase pattern distribution in L2 hindguts. $(D-H)$ Cell cycle markers during Drosophila pupal day 2 (P2) polyploid mitotic cycles. (D) Cyclical CycE accumulation. (E) Early (left cell) and late (right cell) S phases revealed by EdU (green). $\left(F, F^{\prime}\right)$ CycA (green) transitions from cytoplasmic $(F)$ to exclusively nuclear $\left(F^{\prime}\right)$ accumulation in G2 polyploid cells. $\left(F^{\prime \prime}\right)$ CycA levels drastically decline by metaphase. $(G)$ Distribution of various cell cycle marker accumulation during (P2) mitotic cycling. $(H)$ Phospho-tyrosine (phospho-Y) labeling (green) to highlight membranes confirms that rectal papillar cells remain mononucleate following polyploid mitosis (single papilla from young adult). (I) EdU incorporation into distal but not proximal papillar cells $52 \mathrm{~h}$ post-puparium formation. DAPI (magenta) marks nuclei in $A, B, D-F$, $H$, and $I$. Bars: white, $5 \mu \mathrm{m}$; red, $100 \mu \mathrm{m}$. was greatly reduced (Fig. 5E,G). The papillae that did form also contained a somewhat reduced number of cells. Our results suggest that Notch signaling plays a role in reorganizing larval rectal cells into precisely four papillae, and may also influence the transitions between the endocycle and mitotic cycle in these cells, as it does at many other times during development.

\section{Polyploid rectal mitotic cycles are error-prone}

To investigate whether polyploid mitotic divisions are associated with an elevated frequency of chromosome aberrations, we examined mitotic chromosomes stained with DAPI (and, in some cases, $\mathrm{PH} 3$ ) from polyploid rectal papillar cells, and compared them with chromosomes in diploid rectal, ovarian follicle, or embryonic cells. While chromosomes appeared normal in most dividing cells (Fig. 6A,C), we saw an unusually large number of defects in the polyploid divisions. For example, 13 out of $82(15 \%)$ polyploid anaphases from both fixed and live imaging contained lagging chromosomes (Fig. 6B,C), compared with none out of $506(0 \%)$ in diploid embryonic controls (Fig. 6A [inset], C), a highly significant difference $(P \ll$ $0.001)$. PH3 staining frequently persisted on the lagging chromosomes (Fig. 6B).

Fine chromatin bridges constituted a second common type of abnormality (Fig. 6D). When we analyzed dividing cells with normal (Fig. 6E) or bridged (Fig. 6E') chromosomes using live imaging, we observed that the length of anaphase was significantly elongated in the latter group (Fig. 6F). We counted the fraction of polyploid rectal cells
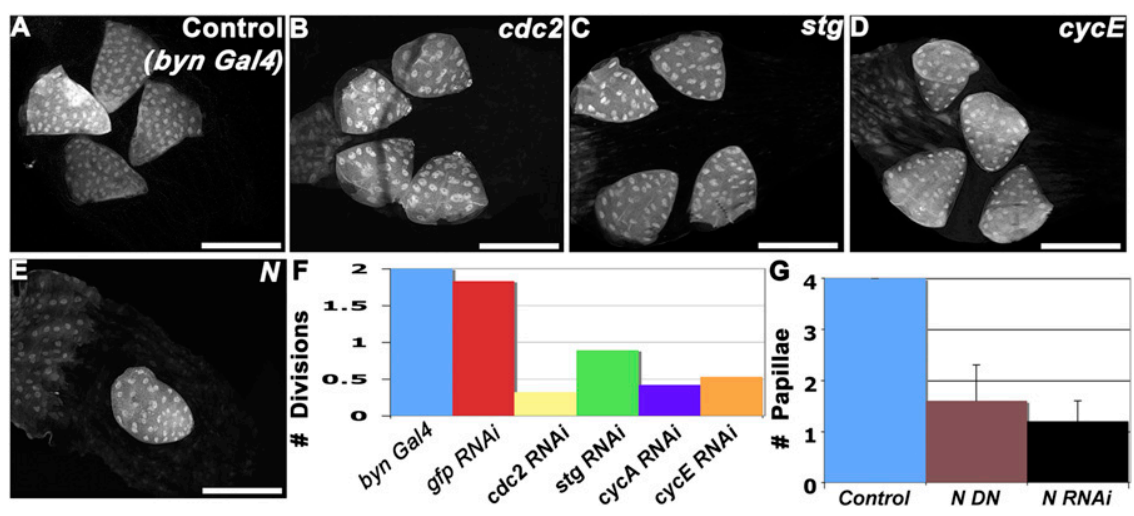

Figure 5. Genetic requirements for polyploid cell proliferation. $(A-E)$ Rectal papillae from adults expressing hindgut targeted transgenes (byn-Gal4, UAS-GFP, UAS ${ }^{-}$) only during pupal development $\left(\mathrm{GAL} 80^{\text {ts }}\right.$ shifted at the end of larval development). (A) Control without RNAi expression. $(B)$ cdc2 RNAi. (C) stg RNAi. (D) cycE RNAi. (E) Similar experiment, but expressing Notch Dominant-Negative. In addition to reduced papillar cell number, only one to two rectal papillae are present instead of four. $(F)$ Summary of disrupted gene expression effects on rectal papillar divisions. The number of divisions required to produce the observed average number of rectal cells per papilla in the experiments of $A-D$ (and with $g f p R N A i$ and $c y c A$ RNAi) based on an initial value of 25 cells per papilla. (G) Notch is required for rectal papillar development. Quantitation of rectal papillar number in control and Notch mutant animals. Bar, $100 \mu \mathrm{m}$. 

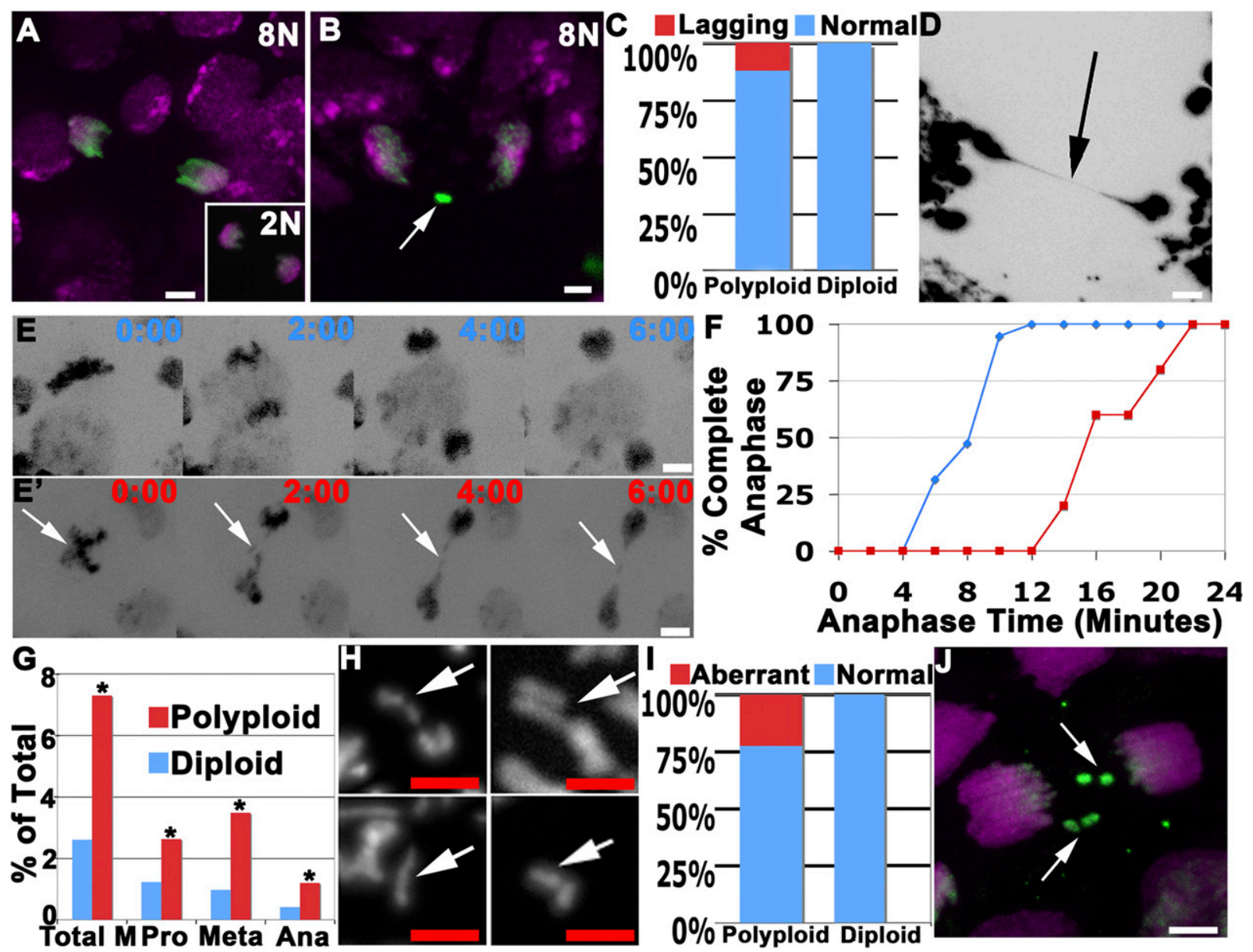

Figure 6. Polyploid mitotic divisions are error-prone. Drosophila pupal rectal cell anaphases show normal chromosome segregation $(A)$ or a lagging chromosome with persistent $\mathrm{PH} 3(B) .(A)$ Normal chromosome segregation and $\mathrm{PH} 3$ labeling in polyploid and diploid (inset) late anaphases. $(B)$ Late anaphase polyploid cell with lagging chromosomes (arrow) and persistent PH3. (C) Elevated frequency $(P>0.001)$ of lagging chromosomes in late anaphases of polyploid papillar cells $(n=82)$ versus diploid embryonic cells $(n=506)$. $(D)$ Fine chromosome bridge (arrow) during polyploid anaphase. $\left(E, E^{\prime}\right)$ Live imaging of normal $(E)$ and aberrant $\left(E^{\prime}\right)$ polyploid anaphases with chromosome bridges (arrows). (F) Delayed anaphase in rectal cells with chromosome bridges (red; $n=5$ ) compared with those without chromosome bridges (blue; $n=19$ ), determined directly from movies. (G) Increased length of mitotic stages in rectal polyploid cells $(n=1756)$ compared with diploid ovarian follicle cells $(n=4654)$ inferred from stage frequency. (Total M) All mitotic stages; (Pro) prophase; (Meta) metaphase; (Ana) anaphase. ${ }^{\star}$ ) Significant to $P<0.001$. (H) Aberrant chromosomes (arrows) from mitotic polyploid cells (for normal chromosomes, see Fig. 2C). (Top two images) Broken chromosomes. (Bottom two images) Acentric fragments. (I) Elevated frequency of chromosome aberrations $(P<.01)$ in polyploid rectal papillae $(n=54)$ versus diploid outer rectal cells $(n=40)$. (J) Culex polyploid late anaphase with multiple lagging chromosomes (arrows) and persistent PH3. DAPI labels chromosomes in $A, B, D$, $H$, and $J$. Dual-labeled images: (green) PH3; (magenta) DAPI. Histone-RFP labels chromosomes in $E$. Bars: white, $5 \mu \mathrm{m}$; red, $1 \mu \mathrm{m}$.

(most of which lacked obvious chromosomal defects) in the various stages of mitosis and compared them with diploid ovarian follicle cells, which divide with a similar half-life (Margolis and Spradling 1995). These data suggested that polyploid rectal cells spend 2.8-fold more time in mitosis than ovarian follicle cells, and that prophase, metaphase, and anaphase all take place more slowly (Fig. 6G).

Finally, we observed a substantial number of additional, more diverse chromosome abnormalities in polyploid papillar cells. These included broken and acentric chromosomes (Fig. 6H). Altogether, $22 \%$ of dividing rectal papillar cells contained such abnormalities $(n=54)$, compared with $0 \%$ of dividing diploid rectal cells $(n=40)$ (Fig. $6 \mathrm{I})$. Anaphase abnormalities were also observed frequently
$(23 \%, n=22)$ in dividing polyploid ileal cells in Culex (Fig. 6J). This argues that chromosome instability is caused by a polyploid mitotic cycle, rather than being a property of rectal papillar physiology.

\section{Discussion}

Polyploid cells can re-enter the mitotic cell cycle

Our results demonstrate that somatic polyploidy is compatible with mitotic proliferation, even though this strategy has not been clearly documented previously during normal development. We found that polyploid Drosophila rectal cells re-enter the mitotic cell cycle despite having previously undergone two or more endocycles. Our 
experiments suggest that at least some polyploid Culex ileal cells also cycle mitotically, rather than undergoing somatic reduction divisions as suggested previously (Berger 1938). Thus, entry into the endocycle during development is not an irreversible step, as had been widely believed. The general characteristics of polyploid mitotic cycles, including cell cycle gene expression and function, appear to be normal. The polyploid cells of mammals, which often increase in size and prevalence under conditions of stress and during aging (Gupta 2000), provide promising material in which to search for other examples.

Polyploid mitotic cycling might be advantageous when a population of differentiated polyploid cells needs to expand only a small amount without changing cell fate. The polyploid Drosophila midgut digestive cells and ileal absorptive cells turn over during pupal development and are replaced by the differentiation of diploid progenitors (Fox and Spradling 2009; Mathur et al. 2010). These new adult tissues are likely better adapted for an adult diet and lifestyle. However, larval and adult rectal cells are both thought to carry out the same function: helping to maintain the water and ion balance of the hemolymph (Bradley 1987). The addition of papillae in adulthood may simply increase rectal cell number and surface area, but not require a change in cell fate. Malpighian tubules also contribute to these same functions, and this polyploid larval tissue also survives into adulthood, but without any change in cell number.

\section{Polyploidy and mitotic fidelity}

Our results suggest a second reason for the rarity of polyploid mitotic cycles during development: This strategy may impose a significant cost by decreasing genomic stability. Polyploid mitotic rectal cells display a greatly elevated frequency of mitotic abnormalities compared with diploid cells, including chromosome bridges, lagging chromosomes, chromosome fragments, and delayed mitotic transits. These observations support the view that polyploidy renders cells inherently less able to divide faithfully (Ganem et al. 2007). Natural polyploid mitotic cells may get away with propagating the genome less faithfully than diploid cells because the cost of making mistakes is lower. Changes in gene copy number resulting from chromosome aberrations would represent a smaller proportional alteration in gene dosage in a polyploid compared with a diploid cell. Indeed, the highly polyploid macronuclei of ciliates divide amitotically, without accurate segregation of sister chromatids (Cervantes et al. 2006).

Growing evidence suggests that alterations in DNA replication are responsible for the genomic instability of polyploid cells. Replication during normal mitotic $S$ phase uses sophisticated mechanisms and checkpoints that ensure high-fidelity duplication of the entire genome. However, the endocycle $S$ phase is often much less faithful (Lee et al. 2009). While "once per cell cycle" initiation is retained, the loss of $M$ phase uncouples the normal replication completion checkpoint, and the checkpoint linking damage to p53-induced apoptosis is also frequently absent (Mehrotra et al. 2008). This loosening of replication controls may facilitate specialized genomic changes-such as chorion gene amplification (Spradling and Mahowald 1980) or genome reprocessing (Prescott 2000) - that are known to occur only in polyploid ovarian follicle cells or polyploid ciliate macronuclei, respectively. A much more common consequence of these changes in replication control is that many polyploid Drosophila cells lack a late S phase and fail to replicate genomic heterochromatin at each endocycle. Even though we observed late $S$ phases during larval rectal endocycles, genome replication may still have been incomplete. Unreplicated regions might generate bridges, lagging chromosomes, and chromosome breaks following re-entry into mitosis. Thus, the genomes of polyploid cells may already contain the seeds of chromosomal damage even prior to re-entering the mitotic cell cycle.

\section{Polyploidy and cancer}

Our results support the view that polyploidy followed by mitosis contributes to the origin of some cancers. Cells mutant for the Bloom's syndrome (BLM) and Fanconi anemia (FANCA-N) genes, syndromes that predispose patients to cancer, display a suite of chromosome abnormalities similar to those reported here for mitotic polyploid cells (Chan et al. 2007, 2009). These include an elevated frequency of anaphases bridges, lagging chromosomes, and micronuclei (containing lost chromosomes). BLM encodes a conserved RecQ helicase that acts at stalled replication forks, while the Fanconi anemia core complex is required for S-phase DNA damage responses and may interact with BLM (Meetei et al. 2003). Cells subjected to replication stress show greatly elevated numbers of "ultrafine DNA bridges" containing BLM protein and associated with FANCD2 protein at each end, which are thought to result from unresolved DNA replication structures (Chan et al. 2009). Thus, utilization of the endocycle may generate the same types of DNA damage as loss of these key replication proteins or a period of replication stress-situations that are known to predispose cells to oncogenic transformation.

Consequently, polyploidy followed by a return to the mitotic cycle may increase the chance that cells will give rise to cancer. Changes in the cytoplasm-for example, the production of extra centrosomes (Fukasawa 2005)—may be partially responsible by inducing aberrant spindles (Ganem et al. 2009). However, our results highlight the importance of learning how genome reduplication changes during developmental endocycles, as well as in polyploid cells induced by environmental factors, stress, and aging. Comparing polyploid rectal cells with other Drosophila polyploid tissues demonstrates that the magnitude of these changes varies between cell types. Some of these replication changes, but not others, may be conducive to gene amplification, a process seen in some polyploid cells and cancers, but whose regulation is poorly understood. Our finding of normal polyploid cells that re-enter the mitotic cycle now provides a highly favorable system in which to address these questions and learn more about 
the origin of mitotic instability during somatic development.

\section{Materials and methods}

Drosophila culture

Drosophila were obtained from the Bloomington Stock Center, and were cultured on standard food at room temperature $\left(21^{\circ} \mathrm{C}-22^{\circ} \mathrm{C}\right)$ unless otherwise indicated. Pupae were collected as white prepupae, which are designated as $0 \mathrm{~h}$ post-prepupae formation.

\section{Mosquito culture}

Mosquito larvae (C. pipiens) were supplied by Carolina Biologicals, and were kept in water supplemented with $1 \mathrm{mM} \mathrm{NaHCO}_{3}, 1 \mathrm{mM}$ $\mathrm{CaCl}_{2}$, and $1 \mathrm{uM}$ EDTA, plus powdered milk. Larvae were monitored every hour for pupation. At appropriate times, pupae were dissected and fixed as for Drosophila (Fox and Spradling 2009).

\section{Antibody staining/EdU labeling}

Hindgut tissue was fixed and stained with primary and secondary antibodies as described previously (Fox and Spradling 2009). The antibodies used were as follows: mouse PH3 (Ser 10) (1:2000; Cell Signaling), rat CycE (1:500; Helena Richardson), guinea pig CycE (1:500; Terry Orr-Weaver), rabbit CycA (1:500; Christian Lehner and Frank Sprenger), rabbit anillin (1:100; Christine Field), mouse phospho-tyrosine (1:1000; Upstate Biotechnologies), mouse anti-Delta (1:100; Developmental Studies Hybridoma Bank), mouse anti-GFP (1:2000; Invitrogen), and Alexa-conjugated IgG secondary antibodies (1:1000; Molecular Probes). DAPI staining was at $5 \mu \mathrm{g} / \mathrm{mL}$, except for ploidy analysis $(50 \mathrm{ng} / \mathrm{mL})$. For EdU analysis, tissue was incubated for $30 \mathrm{~min}$ in $50 \mathrm{mM} \mathrm{EdU}$ (Invitrogen) prior to fixation, and detection was per the manufacturer's protocol.

\section{Microscopy}

Mitotic chromosome and ploidy analysis samples were prepared from Drosophila or Culex hindgut tissue as described by Gatti et al. (1994). Drosophila contained byn-Gal4 and either UASGFP or UAS ds-Red to facilitate the identification of pupal hindgut tissue. Images were acquired using either a Zeiss Axiophot microscope with Apotome software or a Leica SP5 confocal microscope. For live imaging, guts were dissected from flies bearing fluorescent proteins and cultured as described (Prasad et al. 2007). Images were collected using a spinning-disk confocal microscope (PerkinElmer) at a rate of 30-60 frames per hour for up to $3 \mathrm{~h}$, without any detectable decline in tissue health or division. The constructs used were jupiter-gfp (Karpova et al. 2006), moesin-gfp (Edwards et al. 1997), histone-gfp, or histonerfp (Bloomington Stock Center). Movies were analyzed using ImageJ software.

\section{Image analysis}

For ploidy analysis, nuclear DAPI staining was quantitated using the integrated density measure in ImageJ. Ploidy was then calculated by normalizing each nucleus relative to the median DAPI intensity of haploid spermatocytes imaged on the same slide with the same settings (Supplemental Fig. S1A). For Culex, the median value of pyloric cells was set at 2 and served as the internal standard. Cell numbers were determined using "cell counter" (ImageJ). Anaphases were scored as containing lagging chromosomes only if chromosomes were clearly not associated with either group of segregated chromosomes in late anaphase. For statistical comparisons, $\chi^{2}$ analysis was used.

\section{Lineage analysis}

Lineage analysis of the genital disc used doublesex (dsx)-Gal4, which is expressed throughout the larval genital imaginal disc (Robinett et al. 2010), but not in larval hindgut cells. dsx-Gal4 animals were crossed to animals containing a G-TRACE construct (Evans et al. 2009), which induces a UAS-based FLPase to excise a stop cassette, allowing expression of a ubi-Stinger reporter. Thus, animals containing both $d s x-G a l 4$ and G-TRACE permanently express ubi-Stinger in all cells derived from the genital imaginal disc.

\section{RNAi knockdown experiments}

To test the function of genes during rectal papillar mitotic divisions, flies bearing appropriate $U A S$ transgenes were crossed to tubulin-Gal80ts; byn-Gal4, UAS-GFP flies. Progeny were kept at $18^{\circ} \mathrm{C}$, where GAL4 expression is inhibited by active GAL 80 until shifting to $30^{\circ} \mathrm{C}$ as wandering larvae, prior to polyploid mitosis. Shifted animals were dissected as young adults, and the cell number within the rectal papillae was counted. The constructs used were as follows: UAS-stg RNAi (v17760, Vienna Drosophila RNAi Center [VDRC]), UAS-cdc2 RNAi (v41838, VDRC), UAS-cyclin A RNAi (v32421, VDRC), UAS-cyclin E RNAi (TRiP.JF02473, Bloomington Stock Center), UAS-Dominant-Negative Notch (Rebay et al. 1993; Jacobsen et al. 1998), and UAS-Notch RNAi (Sarah Bray, University of Cambridge).

\section{Nomenclature and abbreviations}

Genetic symbols are as described in FlyBase. (N) The haploid number of chromosomes (four for Drosophila; three for Culex); (C) the haploid DNA content. We define a "polyploid" cell as a somatic cell that contains more than the diploid number of chromosome sets. The term "endopolyploid" is equivalent. We define the "endocycle" as any programmed cell cycle in which the genome reduplicates but chromosomes are not segregated into two daughter cells. We define the substages of the endocycle as "endo-S phase," "endomitosis," "endoprophase," "endometaphase," etc. Endocycle stages may or may not encompass all of the normal events of the corresponding stage in a mitotic cell cycle. Polyploid cells generated by external factors such as antispindle or replication drugs are said to have undergone cell cycle errors-i.e., "failed cytokinesis" - rather than an endocycle. It is recognized that, in some cases, the distinction may be difficult to determine.

\section{Acknowledgments}

The following kindly provided reagents: Bruce Baker, Sarah Bray, Christine Field, Dan Kiehart, Christian Lehner, Mary Lilly, Terry Orr-Weaver, Mark Peifer, Helena Richardson, Nasser Rusan, and Frank Sprenger. We thank members of the Spradling and Gall laboratories for useful feedback and technical advice. Jianjun Sun and Joshua Bembenek provided useful comments on the manuscript. D.T.F. is a fellow of the Jane Coffin Childs Memorial Fund for Medical Research. A.C.S. is an investigator with the Howard Hughes Medical Institution.

\section{References}

Berger C. 1938. Multiplication and reduction of somatic chromosome groups as a regular developmental process in the mosquito, Culex pipiens. Contributions embryol 27: 200-232. 
Berridge MJ, Gupta BL. 1967. Fine-structural changes in relation to ion and water transport in the rectal papillae of the blowfly, Calliphora. J Cell Sci 2: 89-112.

Bradley TJ. 1987. Physiology of osmoregulation in mosquitoes. Annu Rev Entomol 32: 439-462.

Brodsky VYa. Uryvaeva IV. 1985. Genome multiplication in growth and development. Cambridge University Press, Cambridge, UK.

Cervantes MD, Coyne RS, Xi X, Yao MC. 2006. The condensin complex is essential for amitotic segregation of bulk chromosomes, but not nucleoli, in the ciliate Tetrahymena thermophila. Mol Cell Biol 26: 4690-4700.

Chan KL, North PS, Hickson ID. 2007. BLM is required for faithful chromosome segregation and its localization defines a class of ultrafine anaphase bridges. EMBO J 26: 3397-3409.

Chan KL, Palmai-Pallag T, Ying S, Hickson ID. 2009. Replication stress induces sister-chromatid bridging at fragile site loci in mitosis. Nat Cell Biol 11: 753-760.

Dej KJ, Spradling AC. 1999. The endocycle controls nurse cell polytene chromosome structure during Drosophila oogenesis. Development 126: 293-303.

Edwards KA, Demsky M, Montague RA, Weymouth N, Kiehart DP. 1997. GFP-moesin illuminates actin cytoskeleton dynamics in living tissue and demonstrates cell shape changes during morphogenesis in Drosophila. Dev Biol 191: 103117.

Evans CJ, Olson JM, Ngo KT, Kim E, Lee NE, Kuoy E, Patananan AN, Sitz D, Tran P, Do MT, et al. 2009. G-TRACE: Rapid Gal4-based cell lineage analysis in Drosophila. Nat Methods 6: 603-605.

Fox DT, Spradling AC. 2009. The Drosophila hindgut lacks constitutively active adult stem cells but proliferates in response to tissue damage. Cell Stem Cell 5: 290-297.

Fukasawa K. 2005. Centrosome amplification, chromosome instability and cancer development. Cancer Lett 230: 6-19.

Ganem NJ, Storchova Z, Pellman D. 2007. Tetraploidy, aneuploidy and cancer. Curr Opin Genet Dev 17: 157-162.

Ganem NJ, Godinho SA, Pellman D. 2009. A mechanism linking extra centrosomes to chromosomal instability. Nature 460: $278-282$.

Gatti M, Bonaccorsi S, Pimpinelli S. 1994. Looking at Drosophila mitotic chromosomes. Methods Cell Biol 44: 371-391.

Graves BJ, Schubiger G. 1982. Cell cycle changes during growth and differentiation of imaginal leg discs in Drosophila melanogaster. Dev Biol 93: 104-110.

Gupta S. 2000. Hepatic polyploidy and liver growth control. Semin Cancer Biol 10: 161-171.

Jacobsen TL, Brennan K, Martinez Arias A, Muskavitch T. 1998. Cis-interactions between Delta and Notch modulate neurogenic signaling in Drosophila. Development 125: 45314540.

Karpova N, Bobinnec Y, Fouix S, Huitorel P, Debec A. 2006. Jupiter, a new Drosophila protein associated with microtubules. Cell Motil Cytoskeleton 63: 301-312.

Lee HO, Davidson JM, Duronio RJ. 2009. Endoreplication: Polyploidy with purpose. Genes Dev 23: 2461-2477.

Mathur D, Bost A, Ohlstein B. 2010. A transient niche regulates the specification of Drosophila intestinal stem cells. Science 327: 210-213.

Margolis J, Spradling A. 1995. Identification and behavior of epithelial stem cells in the Drosophila ovary. Development 121: 3797-3807.

Meetei AR, Sechi S, Wallisch M, Yang D, Young MK, Joenje H, Hoatlin ME, Wang W. 2003. A multiprotein nuclear complex connects Fanconi anemia and Bloom syndrome. Mol Cell Biol 23: 3417-3426.
Mehrotra S, Maqbool SB, Kolpakas A, Murnen K, Calvi BR. 2008. Endocycling cells do not apoptose in response to DNA rereplication genotoxic stress. Genes Dev 22: 3158-3171.

Prasad M, Jang AC, Starz-Gaiano M, Melani M, Montell DJ. 2007. A protocol for culturing Drosophila melanogaster stage 9 egg chambers for live imaging. Nat Protoc 2: 2467-2473.

Prescott DM. 2000. Genome gymnastics: Unique modes of DNA evolution and processing in ciliates. Nat Rev Genet 1: 191-198.

Rajagopalan H, Lengauer C. 2004. Aneuploidy and cancer. Nature 432: 338-341.

Rebay I, Fehon RG, Artavanis-Tsakonas S. 1993. Specific truncations of Drosophila Notch define dominant activated and dominant negative forms of the receptor. Cell 74: 319-329.

Robinett CC, Vaughan AG, Knapp JM, Baker BS. 2010. Sex and the single cell. II. There is a time and a place for sex. PLoS Biol 8: e1000365. doi: 10.1371/journal.pbio.1000365.

Skaer H. 1993. The alimentary canal. In The development of Drosophila melanogaster (ed. M Bate, AMartinez Arias), pp.941-1012. Cold Spring Harbor Laboratory Press, Cold Spring Harbor, NY.

Spradling A, Mahowald A. 1980. Amplification of genes for chorion proteins during oogenesis in Drosophila melanogaster. Proc Natl Acad Sci 77: 1096-1100. 


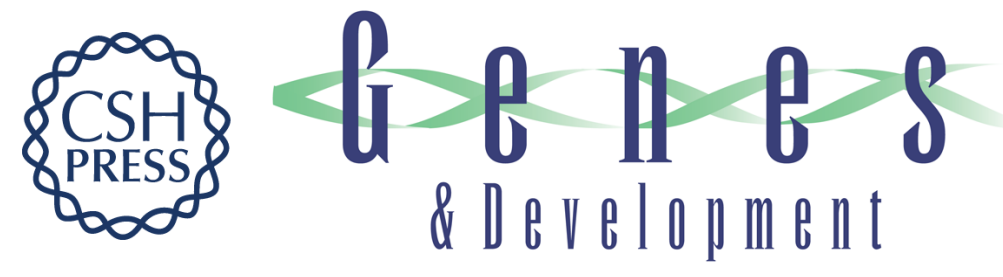

\section{Error-prone polyploid mitosis during normal Drosophila development}

Donald T. Fox, Joseph G. Gall and Allan C. Spradling

Genes Dev. 2010, 24:

Access the most recent version at doi:10.1101/gad.1952710

Supplemental

Material

References

License

Email Alerting Service
http://genesdev.cshlp.org/content/suppl/2010/10/12/24.20.2294.DC1

This article cites 29 articles, 10 of which can be accessed free at: http://genesdev.cshlp.org/content/24/20/2294.full.html\#ref-list-1

Receive free email alerts when new articles cite this article - sign up in the box at the top right corner of the article or click here.

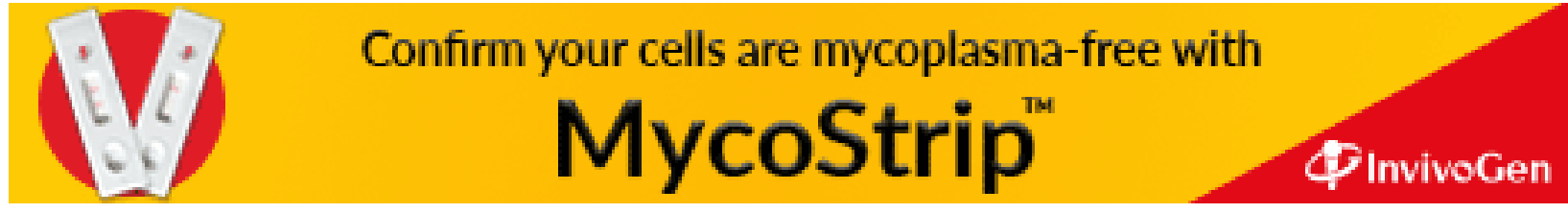

